\title{
ON THE SIEVE METHOD OF VIGGO BRUN
}

\author{
R. D. JAMES
}

1. Introduction. Numerous improvements have been made in the the sieve method since the appearance of Brun's work. ${ }^{1}$ Rademacher, ${ }^{2}$ Estermann, ${ }^{3}$ and, more recently, Buchstab ${ }^{4}$ have introduced new ideas and so obtained more precise results. If their work is examined it will be seen that the various estimates which they use can all be made to depend on the single estimate

$$
\sum_{p \leqq x}(\log p) / p=\log x+O(1),
$$

the summation extending over all primes $p \leqq x$.

In a previous paper ${ }^{5}$ it was shown that Rademacher's results could be extended to any infinite set of primes for which the estimate

$$
\sum_{p \leqq x}^{\prime}(\log p) / p=r \log x+O(1)
$$

applies. Here, and subsequently, the dash indicates that the summation extends over all the primes of a given infinite set which do not exceed $x$, and $r$ is a given real number such that $0<r \leqq 1$.

In this paper it will be shown that Buchstab's results can be similarly extended. Application will be made to primes in arithmetic progression since the set of all primes $p \equiv h(\bmod k)$ with $(h, k)=1$ satisfies (1.2) with $r=1 / \phi(k)$.

2. Preliminary lemmas. It is well known ${ }^{6}$ that from (1.1) it follows that

and

$$
\sum_{p \leqq x} 1 / p=\log \log x+C+O(1 / \log x)
$$

$$
\prod_{p \leqq x}(1-(2 / p))=D / \log ^{2} x+O\left(1 / \log ^{3} x\right),
$$

Presented to the Society, September 5, 1941 under the present title and April 17, 1942 under the title On Euler's conjecture; received by the editors August 26, 1942.

1 Le crible d'Eratosthene et le théorème de Goldbach, Christiania, 1920

2 Abh. Math. Sem. Hamburgischen Univ. vol. 3 (1924) pp. 12-30.

${ }^{3}$ J. Reine Angew. Math. vol. 168 (1932) pp. 106-116.

4 Rec. Math. (Mat. Sbornik) N.S. vol. 46 (1938) pp. 375-387. C. R. (Doklady) Acad. Sci. URSS. vol. 29 (1940) pp. 544-548.

5 Trans. Amer. Math. Soc. vol. 43 (1938) pp. 296-302.

6 Landau, Primzahlen, vol. I, pp. 98-102. 
where $C$ and $D$ are constants. These two estimates form the basis of Rademacher's results.

In precisely the same way it follows from (1.2) that

$$
\sum_{p \leqq x}^{\prime} 1 / p=r \log \log x+C(r)+O(1 / \log x)
$$

and

$$
\prod_{p \leqq x}^{\prime}(1-(2 / p))=D(r) / \log ^{2 r} x+O\left(1 / \log ^{2 r+1} x\right),
$$

where $C(r)$ and $D(r)$ depend only on $r$. Another result which Buchstab uses (Lemma 3 of the first paper referred to) and which also follows from (1.1) is

$$
\begin{aligned}
& \sum_{x^{1 / v} \leqq p<x^{1 / u}} 1 /\left\{p(\log x-\log p)^{2}\right\} \\
& =\left(1 / \log ^{2} x\right)\{\log (v-1)-\log (u-1) \\
& \quad+u /(u-1)-v /(v-1)\}+O\left(1 / \log ^{3} x\right),
\end{aligned}
$$

where $u$ and $v$ are real numbers such that $2 \leqq u \leqq v$.

This may also be written in the form

$$
\begin{aligned}
\sum_{x^{1 / v} \leqq p<x^{1 / u}} 1 /\left\{p(\log x-\log p)^{2}\right\} \\
=\left(1 / \log ^{2} x\right) \int_{u-1}^{v-1}(z+1) z^{-2} d z+O\left(1 / \log ^{3} x\right)
\end{aligned}
$$

which may be generalized.

The proof of (2.4) is made in the usual way by partial summation. An outline of the proof follows. Let

so that by (1.1)

$$
R(x)=\sum_{p \leqq x}(\log p) / p
$$

$$
R(x)=\log x+r(x),
$$

where $r(x)=O(1)$ and, in particular $r(1)=0$. Then the left side of (2.4) is equal to

$$
\begin{aligned}
& \sum\{R(n)-R(n-1)\} /\left\{\log n(\log x-\log n)^{2}\right\} \\
&= \sum\{\log n-\log (n-1)\} /\left\{\log n(\log x-\log n)^{2}\right\} \\
&+\sum\{r(n)-r(n-1)\} /\left\{\log n(\log x-\log n)^{2}\right\}
\end{aligned}
$$

where the summation range is $x^{1 / v} \leqq n<x^{1 / u}$. Since

$$
\log n-\log (n-1)=1 / n+O\left(1 / n^{2}\right)
$$


the first sum will be

$$
\sum 1 /\left\{n \log n(\log x-\log n)^{2}\right\}
$$

plus an error term. The sum (2.6), in turn, is given by

$$
\int_{x^{1 / v}}^{x^{1 / u}} 1 /\left\{t \log t(\log x-\log t)^{2}\right\} d t
$$

plus an error term. The substitution $\log t=(\log x) /(z+1)$ transforms this integral into the integral in (2.4). The proof is then completed by showing that the second sum in (2.5) and the various error terms are all $O\left(1 / \log ^{3} x\right)$.

The generalized form of (2.4) is given in the following lemma.

LEMMA 1. Let $s$ be any positive real number and let $u$ and $v$ be positive real numbers such that $1 \leqq u \leqq v$ if $2 s<1,1<u \leqq v$ if $2 s \geqq 1$.

Then

$$
\begin{aligned}
\sum_{x^{1 / v} \leqq p<x^{1 / u}}^{\prime} 1 /\{p & \left.(\log x-\log p)^{2 s}\right\} \\
& =\left(r / \log ^{2 s} x\right) \int_{u-1}^{v-1}(z+1)^{2 s-1} z^{-2 s} d z+O\left(1 / \log ^{2 s+1} x\right) .
\end{aligned}
$$

The proof is omitted since it follows that given for (2.4) but uses (1.2) instead of (1.1).

3. The first theorem. Next, following Rademacher and Buchstab, we define a function $P_{\omega}(x ; y, r)=P\left(x ; y, r ; \Delta, a ; p_{i}, a_{i}, b_{i}\right)$. Let $\Delta$ and $a$ be given positive integers and let $p_{1}, \cdots, p_{k}$ be the primes not dividing $\Delta$ and less than $y$ of a given infinite set of primes for which (1.2) holds. Let $\omega$ denote a given set of non-negative integers $a_{1}, b_{1} ; \cdots ; a_{k}, b_{k} ; a_{i}<p_{i}, b_{i}<p_{i}$ with $a_{i} \neq b_{i}, i=1, \cdots, k$. Then $P_{\omega}(x ; y, r)$ is defined as the number of integers $n$ which satisfy the conditions

$$
\begin{aligned}
& n \leqq x, \quad n \equiv a(\bmod \Delta), \\
&\left(n-a_{i}\right)\left(n-b_{i}\right) \neq 00\left(\bmod p_{i}\right), \quad i=1, \cdots, k .
\end{aligned}
$$

Since $P_{\omega}(x ; y, r)=P_{\omega}(x ; x, r)$ for $y \geqq x$ we may allow $y$ to be infinite and consider an infinite set of integers $a_{i}, b_{i}$.

It follows as in Rademacher's paper that if $p_{k}$ is the largest prime of the given set less than $x^{1 / \gamma}$ then

$$
P_{\omega}\left(x ; x^{1 / \gamma}, r\right)=P_{\omega}\left(x ; p_{k+1}, r\right)>E x / \Delta-R,
$$

where 


$$
\begin{aligned}
E= & \left\{1-2 \sum_{1 \leqq a \leqq k}^{\prime}\left(1 / p_{a}\right)\right\} \\
& +\sum_{1 \leqq a \leqq k}^{\prime} \sum_{1 \leqq b \leqq k 1}^{\prime}\left(4 / p_{a} p_{b}\right)\left\{1-2 \sum_{1 \leqq c \leqq b}^{\prime}\left(1 / p_{c}\right)\right\}+\cdots
\end{aligned}
$$

and

$$
\begin{aligned}
& k \geqq k_{1} \geqq \cdots \geqq k_{n} \geqq 1, \\
& R=(2 k+1)\left(2 k_{1}+1\right)^{2} \cdots\left(2 k_{n}+1\right)^{2} .
\end{aligned}
$$

From (2.1) and (2.2) it follows that for every $\epsilon>0$ there exists a number $w_{0}=w_{0}(\epsilon)$ such that for all $w \geqq w_{0}$ we have

$$
\begin{aligned}
\sum^{\prime} 1 / p & <r \log (m+\epsilon), \\
\Pi^{\prime}(1-(2 / p)) & >(m+\epsilon)^{-2 r}, \quad w \leqq p<w^{m} .
\end{aligned}
$$

We now define three positive numbers $h, B$, and $B^{\prime}$ which depend on $r$. Thus

$$
h=e^{1 / 4 r}-\epsilon, \quad B=(22 / 17)^{1 / r}-\epsilon, \quad B^{\prime}=(26 / 23)^{1 / r}-\epsilon,
$$

where $\epsilon$ is an arbitrarily small positive number. Buchstab uses these numbers with $r=1$ in the proofs of his first two lemmas which correspond to our Theorem 1.

Following Buchstab we choose $k_{1}=k$ where $p_{k}$ is the largest prime of the given set less than $x^{1 / \gamma}$; for $i \geqq 2$ we choose $k_{i}$ so that $p_{k_{i}}$ is the largest prime in the given set less than $x^{1 / \gamma B h^{i-2}}$. The process is continued until we come to $i=t+1$ where $p_{k_{t+1}}$ is the least prime in the given set for which $p_{k_{t+1}}^{1 / h}<w_{0} \leqq p_{k_{t+1}}$. For $t+1<i \leqq n$ we choose $p_{k_{i}}=p_{k_{t+1}}$.

We denote by $E_{v}(v=1,2, \cdots, n)$ the sum formed by taking from $E$ only those terms for which the subscripts on the $p$ 's exceed $k_{v+1}$. We then write

where

$$
E_{v}=\sum_{i=0}^{2 v+1} E_{v}^{(i)}
$$

$$
E_{v}^{(i)}=\sum_{s=0}^{i} E_{v-1}^{(s)} S_{v}^{(i-s)}
$$

and $S_{v}^{(i-s)}$ is the $(i-s)$ th elementary symmetric function of

$$
2 / p_{m}, m=k_{v+1}+1, k_{v+1}+2, \cdots, k_{v} \text {. }
$$

It follows as in the papers of Rademacher and Buchstab that for 
$v \geqq 2$ we have

$$
E_{v} \geqq E_{v-1} P_{v}-\Phi_{v}
$$

where

and

$$
P_{v}=\prod_{k v+1<i \leqq k v}^{\prime}\left(1-\left(2 / p_{i}\right)\right)
$$

$$
\Phi_{v}=\sum_{s=0}^{2 v-1} E_{v-1}^{(s)} S_{v}^{(2 v+1-s)} .
$$

For $v=1$ we have

$$
E_{1}>P_{1}-S_{1}^{(4)}
$$

and hence using (3.3) and (3.4)

$$
\begin{aligned}
E=E_{n}>P_{1} \cdots P_{n}\left\{1-P_{1}^{-1} S_{1}^{(4)}\right. & -P_{1}^{-1} P_{2}^{-1} \Phi_{2} \\
& \left.-P_{1}^{-1} P_{2}^{-1} P_{3}^{-1} \Phi_{3}-\cdots\right\} .
\end{aligned}
$$

From (3.2) and the values for $B$ and $h$ it follows that

$$
\begin{aligned}
& S_{1}^{(1)}=2 \sum_{r_{2}<a \leqq r_{1}}^{\prime} 1 / p_{a}<2 r \log (B+\epsilon)=2 \log (22 / 17), \\
& S_{v}^{(1)}=2 \sum_{r_{v}+1<a \leqq r_{v}}^{\prime} 1 / p_{a}<2 r \log (h+\epsilon)=2 \log e^{1 / 4}=1 / 2 .
\end{aligned}
$$

Similarly from (3.2) we obtain

$$
P_{1}^{-1}<(22 / 17)^{2}, \quad P_{v}^{-1}<e^{1 / 2} .
$$

These numerical results are exactly the inequalities used by Buchstab to estimate the value of $E$ from (3.5). We may therefore use his calculations and conclude that

$$
P_{\omega}\left(x ; x^{1 / \gamma}, r\right)>0.98\left(c x / \log ^{2 r} x\right)+O\left(x / \log ^{2 r+1} x\right)+O\left(x^{\delta}\right)
$$

where

$$
\begin{aligned}
& c=c(r)=\gamma^{2 r} D(r) / \Delta, \\
& \delta=\{3+2 h / B(h-1)\} / \gamma .
\end{aligned}
$$

Similarly it can be shown that

$$
P_{\omega}\left(x ; x^{1 / \gamma}, r\right)<1.016\left(c x / \log ^{2 r} x\right)+O\left(x / \log ^{2 r+1} x\right)+O\left(x^{\delta^{\prime}}\right)
$$

where

$$
\delta^{\prime}=\left\{2+2 h / B^{\prime}(h-1)\right\} / \gamma
$$


If we now choose $\gamma=\gamma(r)$ so that $\delta$ and $\delta^{\prime}$ are each less than 1 we obtain this theorem.

THEOREM 1. We have

$$
\begin{aligned}
& P_{\omega}\left(x ; x^{1 / \gamma}, r\right)>0.98\left(c x / \log ^{2 r} x\right)+O\left(x / \log ^{2 r+1} x\right), \\
& P_{\omega}\left(x ; x^{1 / \gamma}, r\right)<1.016\left(c x / \log ^{2 r} x\right)+O\left(x / \log ^{2 r+1} x\right)
\end{aligned}
$$

independently of the set $\omega$ of integers $a_{i}, b_{i}$.

4. The second theorem. Corresponding to Buchstab's Theorems 1 and 2 we have this theorem.

THEOREM 2. Suppose that for fixed $r$ there exist functions $f_{i}(\alpha)$ and $F_{k}(\alpha)$ having a finite number of finite discontinuities in the respective ranges $0 \leqq \alpha \leqq B_{1}, 0 \leqq \alpha \leqq B_{2}$, where $B_{1}$ and $B_{2}$ are certain constants such that $\left|B_{1}-B_{2}\right| \leqq 1$. Suppose further that the inequalities

$$
\begin{aligned}
& P_{\omega}\left(x ; x^{1 / \alpha}, r\right)>f_{i}(\alpha)\left(x / \log ^{2 r} x\right)+O\left(x / \log ^{2 r+1} x\right), \\
& P_{\omega}\left(x ; x^{1 / \alpha}, r\right)<F_{k}(\alpha)\left(x / \log ^{2 r} x\right)+O\left(x / \log ^{2 r+1} x\right)
\end{aligned}
$$

hold independently of $\omega$. Then the functions $f_{i+1}(\alpha)$ and $F_{k+1}(\alpha)$ defined by

$$
\begin{gathered}
f_{i+1}(\alpha)=f_{i}(\alpha), \quad F_{k+1}(\alpha)=F_{k}(\alpha), \quad 0 \leqq \alpha<1 ; \\
f_{i+1}(\alpha)=\max \left\{f_{i}(\alpha), f_{i}(\beta)-2 r \int_{\alpha-1}^{\beta-1} F_{k}(z)(z+1)^{2 r-1} z^{2 r} d z\right\}, \\
F_{k+1}(\alpha)=\min \left\{F_{k}(\alpha), F_{k}(\beta)-2 r \int_{\alpha-1}^{\beta-1} f_{i}(z)(z+1)^{2 r-1} z^{2 r} d z\right\}, \\
1 \leqq \alpha \leqq \beta \leqq B_{2} ;
\end{gathered}
$$

also satisfy the inequalities (4.1).

Buchstab's proof for the case $r=1$ proceeds as follows. The difference between $P_{\omega}\left(x ; p_{k+1}, 1\right)$ and $P_{\omega}\left(x ; p_{k}, 1\right)$ is the number of integers $n$ of the form

$$
a_{k}+m p_{k} \quad \text { or } \quad b_{k}+m p_{k}, \quad m=0,1,2, \cdots,
$$

which satisfy the conditions (3.1) for $i=1,2, \cdots, k-1$. This is the same as the number of integers $m$ which satisfy the conditions

$$
\begin{aligned}
& m \leqq\left(x-a_{k}\right) / p_{k}, \quad a_{k}+m p_{k} \equiv a(\bmod \Delta), \\
& \left(m-a_{i}^{\prime}\right)\left(m-b_{i}^{\prime}\right) \not \equiv 0\left(\bmod p_{i}\right), \quad i=1,2, \cdots, k-1,
\end{aligned}
$$

plus the number of integers which satisfy a similar set of conditions 
with $a_{k}$ replaced by $b_{k}$. Hence we have

$$
\begin{aligned}
P_{\omega}\left(x_{i} p_{k+1}, 1\right)= & P_{\omega}\left(x ; p_{k}, 1\right)-P_{\omega_{k}^{\prime}}\left(\left(x-a_{k}\right) / p_{k} ; p_{k}, 1\right) \\
& -P_{\omega_{k}^{\prime \prime}}\left(\left(x-b_{k}\right) / p_{k} ; p_{k}, 1\right) .
\end{aligned}
$$

Now, $P_{\omega_{k}}\left(x / p_{k} ; p_{k}, 1\right)$ counts at most one more integer than $P_{\omega_{k}}\left(\left(x-a_{k}\right) / p_{k} ; p_{k}, 1\right)$, so that we have the recursion formula

$$
\begin{aligned}
P_{\omega}\left(x ; p_{k+1}, 1\right)= & P_{\omega}\left(x ; p_{k}, 1\right)-P_{\omega_{k}}\left(x / p_{k} ; p_{k}, 1\right) \\
& -P_{\omega^{\prime}{ }_{k}}\left(x / p_{k} ; p_{k}, 1\right)+\mu_{k}
\end{aligned}
$$

where $0 \leqq \mu_{k} \leqq 2$. Suppose that $p_{j}, \cdots, p_{k}$ are the primes of the given set between $x^{1 / \beta}$ and $x^{1 / \alpha}$; that is,

$$
p_{j-1}<x^{1 / \beta} \leqq p_{j}<\cdots<p_{k}<x^{1 / \alpha} \leqq p_{k+1} .
$$

By repeated application of (4.2) we obtain

$$
\begin{aligned}
P_{\omega}\left(x ; x^{1 / \alpha}, 1\right)= & P_{\omega}\left(x ; x^{1 / \beta}, 1\right)-\sum_{s} P_{\omega_{s}}\left(x / p_{s} ; p_{s}, 1\right) \\
& -\sum_{s} P_{\omega_{s}^{\prime}}\left(x / p_{s} ; p_{s}, 1\right)+\sum_{s} \mu_{s},
\end{aligned}
$$

where $\sum \mu_{s}=O\left(x^{1 / 2}\right)$. The two sums on the right side of (4.3) are each divided into partial sums defined by

$$
x^{1 /\left(u_{i+1}+1\right)} \leqq p<x^{1 /\left(u_{i}+1\right)},
$$

where

$$
u_{i}=\alpha-1+i(\beta-\alpha) / n,
$$

and $n$ is an integer such that $c_{1} \log x \leqq n \leqq c_{2} \log x$.

For a typical partial sum $T_{i}$ we use (4.1) and obtain

$$
\begin{aligned}
T_{i} \leqq & F_{k}\left(u_{i+1}\right) \sum x /\left\{p(\log x-\log p)^{2}\right\} \\
& +O(x / \log x) \sum 1 /\left\{p(\log x-\log p)^{2}\right\} .
\end{aligned}
$$

By Lemma 1 with $s=1, u-1=u_{i}, v-1=u_{i+1}$ this becomes

$$
\begin{gathered}
T_{i} \leqq F_{k}\left(u_{i+1}\right)\left(x / \log ^{2} x\right) \int_{u_{i}}^{u_{i+1}}(z+1) z^{-2} d z \\
+O\left(x / \log ^{3} x\right) \int_{u_{i}}^{u_{i+1}}(z+1) z^{-2} d z
\end{gathered}
$$

Then we have

$$
\sum_{i=0}^{n-1} F_{k}\left(u_{i+1}\right) \int_{u_{i}}^{u_{i+1}}(z+1) z^{-2} d z=\int_{\alpha-1}^{\beta-1} F(z)(z+1) z^{-2} d z+O(1 / \log x) .
$$


Putting these results together we find that

$$
\begin{aligned}
P_{\omega}\left(x ; x^{1 / \alpha}, 1\right)> & \left(x / \log ^{2} x\right)\left\{f_{i}(\beta)-2 \int_{\alpha-1}^{\beta-1} F(z)(z+1) z^{-2} d z\right\} \\
& +O\left(x / \log ^{3} x\right)
\end{aligned}
$$

which means that

$$
f_{i+1}(\alpha)=f_{i}(\beta)-2 \int_{\alpha-1}^{\beta-1} F(z)(z+1) z^{-2} d z
$$

will serve as a new function in (4.1).

The result for $F_{k+1}(\alpha)$ is obtained in exactly the same way. The proof of Theorem 2 is the same except that we use Lemma 1 with $s=r$ instead of $s=1$.

Since $0 \leqq P_{\omega}\left(x ; x^{1 / \alpha}, r\right) \leqq P_{\omega}\left(x ; x^{1 / \gamma}, r\right)$ for $0 \leqq \alpha \leqq \gamma$ it follows from Theorem 1 that we may take

$$
\begin{aligned}
& f_{0}(\alpha)=\left\{\begin{array}{cll}
0 & \text { for } & 0 \leqq \alpha<\gamma, \\
0.98 c & \text { for } & \alpha=\gamma
\end{array}\right. \\
& F_{0}(\alpha)=1.016 c \text { for } 0 \leqq \alpha \leqq \gamma .
\end{aligned}
$$

Then, using Theorem 2 we compute successively $F_{1}(\alpha), f_{1}(\alpha)$; $F_{2}(\alpha), \ldots$ A numerical example to illustrate the process is given in $\$ 6$.

5. A generalization of the second theorem. Let us consider a given infinite set of primes for which

$$
\sum_{p \leqq x}^{\prime \prime}(\log p) / p=s \log x+O(1),
$$

holds and an infinite subset of this set for which (1.2) is satisfied. We define a function $P_{\omega}(x ; z, s ; y, r)$ similar to $P_{\omega}(x ; y, r)$. Let $p_{1}, \cdots, p_{k}$ be the primes of the given set not dividing $\Delta$ and not exceeding $z$ for which (5.1) holds; let $p_{k+1}, \cdots, p_{l}$ be the primes of the subset not dividing $\Delta$, greater than $z$ and not greater than $y$ for which (1.2) holds. Let $\omega$ denote a given set of non-negative integers $a_{1}, b_{1} ; \cdots ; a_{l} b_{l}$; $a_{i}<p_{i}, b_{i}<p_{i}$ with $a_{i} \neq b_{i}, i=1, \cdots, l$. Then $P_{\omega}(x ; z, s ; y, r)$ is defined as the number of integers $n \leqq x$ satisfying the conditions

$$
n \equiv a(\bmod \Delta) ; \quad\left(n-a_{i}\right)\left(n-b_{i}\right) \not \equiv 0\left(\bmod p_{i}\right), \quad i=1,2, \cdots, l .
$$

THEOREM 3. Suppose that for fixed $r, s, A$ there exist functions $g_{i}(\alpha)$, $G_{k}(\alpha)$ which have a finite number of finite discontinuities in the range $0 \leqq \alpha \leqq A$. Suppose further that the inequalities 


$$
\begin{array}{r}
P_{\omega}\left(x, x^{1 / A}, s ; x^{1 / \alpha}, r\right)>g_{i}(\alpha)\left(x / \log ^{2 s} x\right)+O\left(x / \log ^{2 s+1} x\right), \\
P_{\omega}\left(x ; x^{1 / A}, s ; x^{1 / \alpha}, r\right)<G_{k}(\alpha)\left(x / \log ^{2 s} x\right)+O\left(x / \log ^{2 s+1} x\right), \\
0 \leqq \alpha \leqq A,
\end{array}
$$

hold independently of $\omega$. Then the functions $g_{i+1}(\alpha)$ and $G_{k+1}(\alpha)$ defined by

$$
\begin{array}{rrr}
g_{i+1}(\alpha) & =g_{i}(\alpha), \quad G_{k+1}(\alpha)=G_{k}(\alpha), & 0 \leqq \alpha<1, \\
g_{i+1}(\alpha) & =\max \left\{g_{i}(\alpha), g_{i}(\beta)-2 r \int_{\alpha-1}^{\beta-1} G_{k}(z)(z+1)^{2 s-1} z^{-2 s} d z\right\}, \\
G_{k+1}(\alpha) & =\min \left\{G_{k}(\alpha), G_{k}(\beta)-2 r \int_{\alpha-1}^{\beta-1} g_{i}(z)(z+1)^{2 s-1} z^{-2 s} d z\right\}, \\
& 0 \leqq \alpha \leqq A,
\end{array}
$$

are also functions satisfying (5.2).

The method of proof is again that given by Buchstab but we use Lemma 1 instead of (2.4).

Since $P_{\omega}\left(x ; x^{1 / A}, s ; x^{1 / A}, r\right)$ is the same as the function $P_{\omega}\left(x ; x^{1 / A}, s\right)$ first used, it is clear from Theorem 1 that for $A=\gamma$ we may take

$$
\begin{aligned}
& g_{0}(\alpha)=\left\{\begin{array}{cll}
0 & \text { for } & 0 \leqq \alpha<\gamma \\
0.98 c & \text { for } & \alpha=\gamma
\end{array}\right. \\
& G_{0}(\alpha)=1.016 c \quad \text { for } \quad 0 \leqq \alpha \leqq \gamma
\end{aligned}
$$

where $c=c(s)$ is now given by

$$
c=\gamma^{2 s} D(s) / \Delta
$$

instead of (3.6).

6. Some numerical results. An interesting question is that of determining how small $r$ must be in order that there should exist integers $n \leqq x, n \equiv a(\bmod \Delta)$ for which

$$
\left(n-a_{i}\right)\left(n-b_{i}\right) \not \equiv 0\left(\bmod p_{i}\right)
$$

for every $p_{i}$ belonging to the given infinite set satisfying (1.2). Clearly, (6.1) holds if $p_{i}>x$ so that we have to determine values of $r$ for which $P_{\omega}(x ; x, r)>0$.

We shall show that this is true for all sufficiently large $x$ when $r=1 / 5$. For this value of $r$ we find that

$$
h=3.49, \quad B=3.62, \quad B^{\prime}=1.84, \quad \gamma=3.78 .
$$


Then by Theorems 1 and 2 with

$$
f_{0}(3.78)=0.98 c, \quad F_{0}(3.78)=1.016 c
$$

we have

$$
f_{1}(1)=f_{0}(3.78)-(2 / 5) F_{0}(3.78) \int_{0}^{2.78} z^{-2 / 5}(z+1)^{-3 / 5} d z .
$$

It is easy to show that

$$
\int_{0}^{2.78} z^{-2 / 5}(z+1)^{-3 / 5} d z<2.36
$$

and hence

$$
f_{1}(1)>c(1 / 5)\{0.98-(0.4)(1.016)(2.36)\}>0 .
$$

It follows that $P_{\omega}(x ; x, 1 / 5)>0$ for all sufficiently large $x$.

A deeper problem is that of determining the greatest value of $r$ for which $P_{\omega}(x ; x, r)>0$ independently of $\omega$. All that we have been able to prove so far, however, is what we have just shown, namely that $r \geqq 1 / 5$.

As a simple numerical example of the use of Theorem 3 we take $r=1 / 2, s=1$. Here we can improve Theorem 1 by using the results in Buchstab's second paper. From them it follows that we may take

$$
\begin{aligned}
& g_{0}(\alpha)=\left\{\begin{array}{cll}
0 & \text { for } & 0 \leqq \alpha<7, \\
43.34 b & \text { for } & \alpha=7,
\end{array}\right. \\
& G_{0}(\alpha)=56.23 b \quad \text { for } \quad 0 \leqq \alpha \leqq 7 \text {, }
\end{aligned}
$$

where $b=0.4161 \cdots$. Then using Theorem 3 with $r=1 / 2, s=1$ we find successively

$$
G_{1}(6)=50.75 b, \quad g_{1}(6)=32.39 b, \quad G_{2}(5)=47.94 b,
$$

and finally

$$
g_{2}(4)=g_{1}(6)-G_{2}(5) \int_{3}^{5}(z+1) z^{-2} d z>0 \text {. }
$$

We have thus shown that $P_{\omega}\left(x ; x^{1 / 7}, 1 ; x^{1 / 4}, 1 / 2\right)>0$ for all sufficiently large $x$ independently of $\omega$.

Suppose now that we take the set of all odd primes as our set with $s=1$ and the set of all primes congruent to $3(\bmod 4)$ as the subset with $r=1 / \phi(4)=1 / 2$. Since the function $P_{\omega}$ does not decrease if we remove the restriction $a_{i} \neq b_{i}$ we may choose 


$$
\begin{array}{cc}
a_{i}=0, \quad b_{i} \equiv x\left(\bmod p_{i}\right), & 0 \leqq b_{i}<p_{i}, \\
x \equiv 2(\bmod 4), \quad & a=1, \quad \Delta=4 .
\end{array}
$$

Then $P_{\omega}\left(x ; x^{1 / 7}, 1 ; x^{1 / 4}, 1 / 2\right)$ counts the number of integers $n \leqq x$ such that

$$
n \equiv 1(\bmod 4), \quad n(n-x) \not \equiv 0\left(\bmod p_{i}\right)
$$

where $p_{1}, \cdots, p_{k}$ are the primes $3,5, \cdots$ up to $x^{1 / 7}$, and $p_{k+1}, \cdots, p_{l}$ are the primes from $x^{1 / 7}$ to $x^{1 / 4}$ which are congruent to $3(\bmod 4)$. It follows that neither $n$ nor $x-n$ is divisible by any prime up to $x^{1 / 7}$ nor by any prime congruent to $3(\bmod 4)$ up to $x^{1 / 4}$.

Now let $M$ and $N$ be the number of primes congruent to 1 and 3 $(\bmod 4)$, respectively, which divide $n$, prime factors of multiplicity $m$ counting $m$ times. Each prime of the first set exceeds $x^{1 / 7}$ and each prime of the second exceeds $x^{1 / 4}$. Since $n \leqq x$ the product of primes dividing $n$ cannot exceed $x$. Hence we have

$$
M / 7+N / 4<1 \text {. }
$$

Furthermore, $n \equiv 1(\bmod 4)$ and so $N$ must be even. The only possible integral solutions of (6.2) are then

$$
\begin{array}{ll}
N=0, & M \leqq 6 ; \\
N=2, & M \leqq 3 .
\end{array}
$$

Since $x \equiv 2(\bmod 4)$ the same reasoning applies to $x-n$. We have thus shown that every sufficiently large integer $x \equiv 2(\bmod 4)$ is the sum of two integers which either have at most six prime factors all congruent to $1(\bmod 4)$ or else have exactly two prime factors congruent to $3(\bmod 4)$ and at most three congruent to $1(\bmod 4)$.

It seems likely that a slight improvement in the method of proof would lead to the elimination of the second possibility, but at present there are still difficulties to be overcome.

UNIVERSITY OF SASKatcheWAN 